\title{
RESIDUOS CERÁMICOS PARA SU POSIBLE USO COMO MATERIA PRIMA EN LA FABRICACIÓN DE CLIINKER DE CEMENTO PORTLAND: CARACTERIZACIÓN Y ACTIVACIÓN ALCALINA
}

\author{
CERAMIC WASTES AS RAW MATERIALS IN PORTLAND CEMENT CLINKER \\ FABRICATION: CHARACTERIZATION AND ALKALINE ACTIVATION
}

\author{
F. PUERTAS ${ }^{(*)}$, A. BARBA(*), M. F. GAZULLA(*), M. P. GÓMEZ(**), M. PALACIOS ${ }^{(*)}$, S. MARTÍNEZ-RAMÍREZ $Z^{(*)}$
}

\section{RESUMEN}

La industria cementera mundial está buscando vías experimentales que permitan desarrollar cementos que precisen menor energía en su formación, degraden menos los entornos y emitan menos gases contaminantes a la atmósfera. Esta línea, en España y Europa, coincide plenamente con el concepto de sostenibilidad y alcanzar el cumplimiento del Protocolo de Kioto. El empleo de diferentes residuos y subproductos industriales como materiales alternativos en la fabricación de cemento se ha revelado como un medio muy efectivo para alcanzar, en gran parte, esa sostenibilidad. Estos materiales alternativos pueden ser incorporados en el proceso de fabricación de cemento, por la sustitución parcial de los combustibles convencionales y/o de las materias primas del crudo y por la incorporación de adiciones activas al cemento y/o clínker.

En el presente trabajo se exponen los resultados preliminares obtenidos al estudiar la composición y activación alcalina de seis residuos cerámicos cocidos con vistas a su viabilidad de sustituir, parcialmente, algunas materias primas, utilizadas en la preparación de un crudo de cemento.

Los resultados obtenidos han mostrado que los seis residuos tienen una composición química y mineralógica adecuada para su posible empleo como materia prima alternativa de un crudo de cemento Portland. No son ni tóxicos ni radioactivos. Como era de esperar estos residuos tienen capacidad puzolánica. A la edad de estudio ( 8 días) la activación alcalina de los residuos es baja. El contenido en fase amorfa y la naturaleza del activador no parecen ser factores que influyan en dicho proceso de activación. El estudio de caracterización realizado sobre las pastas activadas ha demostrado que las fases feldespáticas son las más susceptibles de disolución y reacción con las disoluciones alcalinas.

Palabras clave: fabricación de cemento, residuos cerámicos, caracterización, activación alcalina y puzolanicidad.

\begin{abstract}
SUMMARY
The world-wide cement industry is seeking experimental avenues that will lead to cement production that is less energy-intensive, less damaging to the surrounding environment and less prolific in GHG emissions. In Spain and Europe in general, this approach is wholly consistent with the concept of sustainability and compliance with the Kyoto Protocol. The use of different kinds of industrial waste and by-products as alternative materials in cement manufacture has proved to be a very effective way of contributing to such sustainability. These alternative materials may be incorporated during cement production by partially replacing conventional fuel and/or the raw materials ground into the raw mix, or by including active additions to the cement and/or clinker.
\end{abstract}

The present paper discusses the preliminary results obtained in a study of the composition and alkali activation of six types of fired ceramic waste with a view to their viability as partial replacements for some of the prime materials used to prepare raw mix for Portland cement.

The results show that the chemical and mineralogical composition of the six materials makes them apt for such possible use. They are neither toxic nor radioactive. And as might be expected, this type of waste exhibits pozzolanic activity. Alkali activation of the waste, low at the age analyzed (8 days), does not appear to be affected by the vitreous phase content or the nature of the activator. The characterization study conducted on the activated paste shows that the feldspar phases are the ones most susceptible to dissolution and reaction with the alkaline solutions.

Keywords: cement manufacture, ceramic waste, characterization, alkali activation, pozzolanicity.

\footnotetext{
(*) Instituto de Ciencias de la Construcción Eduardo Torroja (CSIC). Madrid (ESPAÑA).

${ }^{(* *)}$ Instituto de Tecnología Cerámica. Asociación de Investigación de las Industrias Cerámicas. Universitat Jaume I. Castellón (ESPAÑA). Persona de contacto/Corresponding author: puertasf@ietcc.csic.es (F. Puertas).
} 


\section{INTRODUCCIÓN}

La fabricación de cemento Portland se inicia con la preparación de un crudo a partir de unas materias primas determinadas (calizas y margas o arcillas junto con otros modificadores de módulos como cenizas de piritas, arena, etc.), que en sus dosificaciones adecuadas constituyen el crudo de cemento. Este crudo es introducido, de manera continua, a través de un precalcinador a un horno rotatorio cilíndrico, donde el crudo permanece unos 20-30 minutos a temperaturas entre $850-1.500^{\circ} \mathrm{C}$, siendo calcinado y obteniéndose un mineral denominado clínker. Después del enfriamiento, este clínker es molido con un regulador de fraguado (yeso, generalmente) y con otros materiales (calizas, adiciones, etc.) generando el producto final o cemento.

La fabricación de cemento Portland implica un importante consumo de energía térmica y eléctrica, ya que se precisan muy altas temperaturas $\left(\sim 1.500^{\circ} \mathrm{C}\right)$ para que se completen todas las reacciones químicas que conducen a la formación del clínker del cemento Portland. Debido a las mejoras introducidas en las fábricas, la energía específica requerida se ha visto sensiblemente reducida en los últimos años. Entre 1973 y 1988 la energía específica necesaria para producir clínker disminuyó desde $4.750 \mathrm{MJ} / \mathrm{t}$ de clínker a $3.750 \mathrm{MJ} / \mathrm{t}$. Desde entonces, la energía específica se ha mantenido más o menos constante. Adicionalmente, la industria cementera es también una industria contaminante, ya que explota recursos naturales (canteras) y emite a la atmósfera una gran cantidad de gases contaminantes $\left(\mathrm{CO}_{2}, \mathrm{SO}_{2}, \mathrm{NO}_{x}\right)$. Las emisiones de $\mathrm{CO}_{2}$ están asociadas a la descarbonatación de las calizas que es el constituyente mayoritario del crudo de cemento (superando el $60 \%$ de la emisión total). Los restantes gases contaminantes son emitidos durante la combustión de los combustibles fósiles utilizados en las plantas cementeras.

La industria cementera mundial está buscando vías experimentales que permitan desarrollar cementos que precisen menor energía en su formación, degraden menos los entornos y emitan menos gases contaminantes a la atmósfera. Concretamente en España y Europa esta línea coincide plenamente en conseguir una mayor sostenibilidad y alcanzar el cumplimiento del Protocolo de Kioto.

El empleo de diferentes residuos y subproductos industriales como materiales alternativos en la fabricación de cemento se ha revelado como un medio muy efectivo para alcanzar, en gran parte, esa sostenibilidad (1). Estos materiales alternativos pueden ser incorporados en el proceso de fabricación de cemento, por la sustitución parcial de los combustibles convencionales (2) y/o de las materias primas del crudo y por la incorporación de adiciones activas al cemento y/o clínker. Este último aspecto está ampliamente recogido en las normativas nacionales y europeas relativas al cemento (3).

Respecto al empleo de materias primas alternativas en la fabricación de cemento, en la bibliografía se recoge el estudio de diferentes residuos y subproductos industriales utilizados para dicho fin. Concretamente, escorias cristalizadas de horno alto (4-7), cenizas de incineradora $(8-10)$, cenizas volantes $(11-12)$, residuos de reciclado de aluminio (13-15), residuos con fosfatos (16-18) o lodos de papeleras (19).

En los procesos de producción de baldosas cerámicas se generan varios tipos de residuos industriales, los cuales se pueden clasificar en: residuos procedentes de la depuración de efluentes líquidos (las aguas residuales del proceso), residuos procedentes de la depuración de emisiones gaseosas, residuos sólidos. Los residuos sólidos pueden proceder de las materias primas y de baldosas cerámicas defectuosas antes de ser cocidas (en este caso se denominan residuos crudos) o de baldosas cerámicas defectuosas tras la cocción (en este caso se denominan residuos cocidos) (20-22).

El sector español de fabricantes de baldosas cerámicas ha alcanzado una producción superior a los seiscientos millones de metros cuadrados en los últimos años, situándose entre los cuatro primeros productores del mundo (23-24). Esto puede suponer la generación de alrededor de 50.000 toneladas de residuos cocidos anuales. Desde un punto de vista exclusivamente técnico es posible reutilizar estos residuos cocidos en el propio proceso de producción de baldosas (25-27), consiguiéndose la condición de vertido cero. Sin embargo, su reutilización suele requerir una molturación en instalaciones especiales, implicando unos costes de mantenimiento y gestión que no suelen quedar compensados por el posible ahorro en el consumo de materias primas. En consecuencia, la mayor parte de los residuos cocidos se depositan actualmente en vertederos controlados (28), asumiendo las industrias productoras los costes de la gestión de los vertidos. Aunque los residuos cocidos son químicamente inertes, su almacenamiento en vertederos controlados genera un impacto medioambiental notable dado que el sector de fabricantes de baldosas cerámicas se encuentra muy concentrado geográficamente.

En la bibliografía existen algunos estudios centrados en determinar la capacidad puzolánica de los residuos cerámicos cocidos, así como su posible utilización como materiales de adición a cementos, morteros y hormigones (29-32), pero no se ha encontrado ninguna referencia a su posible uso como materia prima en la fabricación de clínker de cemento. En consecuencia, en este artículo se muestran los resultados preliminares obtenidos al estudiar la composición y activación alcalina de algunos residuos cerámicos cocidos con vistas a su viabilidad de sustituir, parcialmente, algunas materias primas, utilizadas en la preparación de un crudo de cemento.

\section{MATERIALES}

Se han seleccionado seis residuos cocidos, procedentes de diferentes plantas industriales dedicadas a la fabricación de baldosas cerámicas para revestimiento. Se han elegido baldosas de revestimiento porque en la composición de los residuos cocidos interviene el $\mathrm{CaO}$ en el intervalo 7$10 \%$ en peso. En consecuencia, este residuo cocido permitiría introducir $\mathrm{CaO}$ en la formulación de los crudos sustituyendo parcialmente a las materias primas habituales que introducen calizas $y$, de esta forma, se verían disminuidas 
las emisiones de $\mathrm{CO}_{2}$ durante la formación a alta temperatura del clínker, aspecto medioambiental de gran trascendencia y vinculado directamente con las directrices del Protocolo de Kioto.

En particular, se han seleccionado tres residuos cocidos de baldosas fabricadas con arcillas rojas, con un contenido de $\mathrm{Fe}_{2} \mathrm{O}_{3}$ superior al $2 \%$, otros dos residuos cocidos de baldosas fabricadas con arcillas blancas, con un contenido de $\mathrm{Fe}_{2} \mathrm{O}_{3}$ inferior al $2 \% \mathrm{y}$, finalmente, un sexto residuo cocido procedente de la mezcla de residuos blancos y rojos como consecuencia de que hay fabricantes que no gestionan la separación de residuos procedentes de distintos tipos de productos. En la Tabla 1 se detallan las referencias utilizadas para designar a estos seis residuos cocidos.

De cada residuo cocido se han recogido $100 \mathrm{~kg}$ representativos de la totalidad de la producción de cada planta industrial de revestimiento. Todos los residuos cocidos se trituraron en un triturador de mandíbulas, a continuación se realizó un cuarteo y se molturaron en un molino de martillos utilizando un tamiz de $1 \mathrm{~mm}$ de luz de malla. Seguidamente se procedió a un cuarteo para tomar muestras representativas de todo el material en estudio. La fracción de muestra utilizada para la caracterización química y mineralógica se molturó en un molino de anillos de carburo de wolframio.

\section{TÉCNICAS EXPERIMENTALES Y ENSAYOS REALIZADOS}

La caracterización química para la determinación de Si, $\mathrm{Al}, \mathrm{Fe}, \mathrm{Na}, \mathrm{K}, \mathrm{Ca}, \mathrm{Mg}, \mathrm{Ti}, \mathrm{Mn}, \mathrm{P}, \mathrm{S}$ y $\mathrm{Zr}$; se ha realizado mediante espectrometría de fluorescencia de rayos $X$ (FRX) (33-38), utilizando un espectrómetro de FRX, modelo PW 2400, de la firma Philips, provisto de un automuestreador PW 2540 VTC Sample Changer. La preparación de la muestra para la medida por FRX se ha llevado a cabo en forma de discos fundidos (perlas) y de pastillas prensadas. Las perlas se han preparado de forma automática con una perladora modelo PERL X'3 de la firma Philips. Para la calibración y la validación de las medidas por FRX se han utilizado los siguientes materiales de referencia certificados: BCS-CRM 348 Ball Clay, CAS 5 Ball Clay, GBW 03102 Clay, GBW 03118 Graphite ore, GBW 03122 Kaolin, GBW 03123 Wollastonite, BCS-CRM 389 High purity magnesite, BCS-CRM 682/1 Iron ore, BCS-CRM 394 Calcined bauxite, BCS-CRM 201a Nepheline syenite, BCS-CRM 267 Silica brick, IPT-53 Feldespato potásico y SRM 98b.

La caracterización química para la determinación de $\mathrm{Pb}$, $\mathrm{Zn}, \mathrm{Ba}, \mathrm{Cr}, \mathrm{B}, \mathrm{V}, \mathrm{Co}, \mathrm{Ni}, \mathrm{Cu}, \mathrm{As}, \mathrm{Sb}$ y $\mathrm{Cd}$ se ha realizado mediante espectrometría óptica de plasma acoplado inductivamente (ICP-OES) (33-38), utilizando un espectrómetro de emisión atómica, modelo Direct Reading Echelle, de la firma Leeman Labs Inc. La digestión de las muestras se ha realizado con agua regia en caliente. La construcción de las curvas de calibrado se ha llevado a cabo con disoluciones de $1.000 \mathrm{mg} \cdot \mathrm{L}^{-1}$ de cada uno de los elementos de Fluka, y para la validación se utilizaron disoluciones de $1.000 \mathrm{mg} \cdot \mathrm{L}^{-1}$ de cada uno de los elementos de Merck.

La caracterización cristalográfica se ha realizado mediante difracción de rayos $X(D R X)$, utilizando un difractómetro de rayos X (33-38), Theta-Theta modelo D8 Advance, de la firma Bruker. La identificación de sustancias cristalinas se ha realizado mediante la observación de los picos de difracción (posición, intensidad, forma del perfil del difractrograma y fondo de éste) y la comparación con la tabla de patrones catalogada. La cuantificación de las fases cristalinas identificadas se ha realizado mediante el método RIR (reference intensity ratios) (38).

La determinación de la curva de contracción-temperatura (39) se ha realizado mediante un ensayo de fusión en un microscopio de calefacción, modelo Misura-3, de la firma Expert Systems. La preparación de la muestra consiste en conformar, por prensado, un cilindro de 3 $\mathrm{mm}$ de diámetro y $3 \mathrm{~mm}$ de altura, que se aloja en el microscopio de calefacción y se somete a un ciclo de calentamiento con una velocidad de $25^{\circ} \mathrm{C} / \mathrm{min}$, visualizando la evolución de la muestras (y su silueta o contorno) con la temperatura. Mediante un tratamiento de análisis de imagen se determina la evolución de la

TABLA 1

Residuos cocidos estudiados

\begin{tabular}{|c|c|}
\hline REFERENCIA & PROCEDENCIA DEL RESIDUO COCIDO \\
\hline R-R1 & $\begin{array}{c}\text { Fábrica de baldosas para revestimiento con arcillas } \\
\text { rojas }\end{array}$ \\
\hline R-R2 & $\begin{array}{l}\text { Fábrica de baldosas para revestimiento con arcillas } \\
\text { rojas }\end{array}$ \\
\hline R-R3 & $\begin{array}{l}\text { Fábrica de baldosas para revestimiento con arcillas } \\
\text { rojas }\end{array}$ \\
\hline R-B1 & $\begin{array}{l}\text { Fábrica de baldosas para revestimiento con arcillas } \\
\text { blancas }\end{array}$ \\
\hline R-B2 & $\begin{array}{l}\text { Fábrica de baldosas para revestimiento con arcillas } \\
\text { blancas }\end{array}$ \\
\hline R-M & $\begin{array}{l}\text { Fábrica de baldosas para revestimiento con arcillas } \\
\text { rojas y blancas, y sin gestión de residuos. }\end{array}$ \\
\hline
\end{tabular}


contracción de la muestra con la temperatura y se definen los siguientes puntos característicos: $T_{\text {IC }}$ (temperatura inicio de contracción), $T_{F C}$ (temperatura de final de contracción), $T_{R}$ (temperatura de reblandecimiento), $T_{E}$ (temperatura de formación de esfera), $T_{1 / 2}$ (temperatura de formación de semiesfera) y $T_{F}$ (temperatura de fusión).

La toxicidad de los residuos cocidos se ha determinado evaluando la toxicidad de los correspondientes lixiviados. La lixiviación se ha llevado a cabo (40) manteniendo cada muestra en suspensión acuosa y en agitación durante

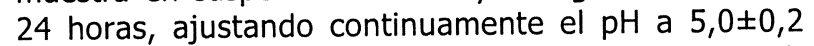
con una disolución de ácido acético al 0,5\%; a continuación se separa la fracción líquida mediante centrifugación y posterior filtración a vacío. La toxicidad de esta fracción líquida se ha determinado mediante el bioensayo de luminiscencia de la bacteria Photobacterium phosphoreum (Vibrio fischeri) (41), utilizando un luminómetro modelo Optocomp 1 de la firma MGM Instruments. El resultado de la determinación de la toxicidad se expresa mediante un parámetro denominado $\mathrm{EC}_{50}$ (que indica la concentración de muestra necesaria para que la luminiscencia de las bacterias se reduzca a la mitad en un período de tiempo de 15 minutos a la temperatura de $15^{\circ} \mathrm{C}$ ) considerándose que un material es tóxico si su lixiviado presenta un valor de $\mathrm{EC}_{50}$ inferior o igual a $3.000 \mathrm{mg} \cdot \mathrm{L}^{-1}$ (39).

La radioactividad de cada residuo cocido se ha determinado mediante un análisis isotópico Gamma utilizando el equipo Ge(HP) ORTEC 919E de la firma Ortec. De cada residuo cocido se ha medido la actividad de los isótopos $\mathrm{Ra}-226$, Th-232 y K-40, y se ha calculado el índice de concentración de actividad definido por la Unión Europea (42). Según este documento cualquier material con un índice de actividad inferior a 6 se puede considerar como no radioactivo.

Se determinó la actividad puzolánica de los residuos, es decir, su capacidad de combinación y reacción con el $\mathrm{Ca}(\mathrm{OH})_{2}$ generado en la hidratación de los silicatos cálcicos del cemento. Esta valoración se realizó siguiendo una doble metodología:

a) De acuerdo con la norma EN 196-5 (43), se utilizó un cemento tipo I $42.5 \mathrm{R}$ y una relación cemento/residuo de 70/30. La preparación y conservación de las mezclas se realizó de acuerdo a la norma, así como la determinación de la concentración de $\mathrm{CaO}$ y $[\mathrm{OH}]$. Estas determinaciones se realizaron a los 8,15 y 28 días de curado.

b) Estudio de la actividad puzolánica mediante disolución saturada de $\mathrm{Ca}(\mathrm{OH})_{2}(44)$. Para ello se prepararon mezclas de $75 \mathrm{ml}$ de la disolución $\mathrm{Ca}(\mathrm{OH})_{2}$ saturada y $1 \mathrm{~g}$ del residuo sólido. Estas mezclas se conservaron, en recipientes herméticamente cerrados, en estufa a $40^{\circ} \mathrm{C}$ hasta las edades de ensayo: 8,15 y 28 días; tras las cuales se determinó la concentración de $\mathrm{CaO}$ y $[\mathrm{OH}]^{-}$por el procedimiento indicado en la norma EN 196-5 (43).

También se estudió la capacidad de esos residuos de formar compuestos tipo geopolímericos por activación alcalina (45). Para ello se prepararon probetas prismáticas de pasta de $1 \times 1 \times 6 \mathrm{~cm}$. Se emplearon dos disoluciones activadoras distintas: $\mathrm{NaOH}$ y waterglass $\left(\mathrm{Na}_{2} \mathrm{O} \cdot \mathrm{nSiO}_{2} \cdot \mathrm{mH}_{2} \mathrm{O}+\mathrm{NaOH}\right)$, con dos concentraciones para cada disolución: 6 M y 12 M. La relación líquido/ sólido fue de 0,4 cuando la disolución activadora era $\mathrm{NaOH} 6 \mathrm{M}$, y 0,5 con los restantes activadores.

A través de ensayos previos, se decidió que el curado de las pastas fuera el siguiente: las primeras $24 \mathrm{~h}$ las probetas se conservaron en estufa a $85^{\circ} \mathrm{C}$ con una H.R. del $99 \%$. El resto del tiempo hasta los 8 días, las probetas se mantuvieron en la cámara de humedad a $99 \%$ H.R. y $21 \pm 2{ }^{\circ} \mathrm{C}$. Tras este tiempo las probetas fueron ensayadas a flexotracción y compresión. Sobre restos de estas muestras se realizó una caracterización a través de DRX, FTIR y ${ }^{29} \mathrm{Si}$ RMN.

El equipo de difracción de rayos $X$ utilizado fue un Philips PW 1730 que consta de un generador de alta tensión de $3 \mathrm{~kW}$, tubo de rayos $\mathrm{X}$ con ánodo de $\mathrm{Cu}$, rendija automática de divergencia y monocromador de grafito. El registro se realizó en el intervalo de $2 \theta$ comprendido entre 5 y $60^{\circ}$. Las pastillas para ser registradas se prepararon con la muestra en polvo, siendo prensadas a una presión de 6 atm, asegurándose que la superficie sobre la que iba a incidir el haz de rayos $X$, estaba perfectamente plana.

Los espectros IR se obtuvieron a través de un espectrómetro marca ATIMATTSON, Génesis series FIIR-TM. Las muestras se prepararon por el método del comprimido de $\mathrm{KBr}$. En la preparación de las muestras se utilizó una cantidad de muestra problema entre $1,0-1,2 \mathrm{mg}$ y 300 $\mathrm{mg}$ de $\mathrm{KBr}$. El barrido de frecuencias se realizó en el rango de $4.000-400 \mathrm{~cm}^{-1}$.

Los registros de RMN se obtuvieron en un equipo marca BRUKER MSL 400. Las condiciones de registro para ${ }^{29} \mathrm{Si}$ fueron: frecuencia de resonancia $79,49 \mathrm{MHz}$, número de adquisiciones de señal por muestra 800 , tiempo de relajación de la señal $5 \mathrm{~s}$, velocidad de rotación de la muestra 4 $\mathrm{KHz}$. La señal química obtenida se midió en ppm, tomando como referencia la señal del trimetil-silano (TMS).

\section{RESULTADOS Y DISCUSIÓN}

Entre las principales características que debe de reunir un residuo para su posible incorporación a un crudo de cemento, están, por una parte, una composición química y mineralógica adecuada y, por otra, una muy baja o nula toxicidad. A continuación se presentan los resultados obtenidos al estudiar estas características en los residuos.

En las Tablas 2 y 3 se muestra la composición química de los seis residuos cocidos: tanto los elementos químicos habituales en los productos cerámicos tradicionales (Tabla 2) como aquellos elementos que pueden aparecer como contaminaciones (Tabla 3). Asimismo, en la Tabla 2 se muestra la pérdida de masa (ppc) de cada residuo cocido cuando se calienta a $1.025^{\circ} \mathrm{C}$. Puede observarse que los cinco residuos cocidos están constituidos fundamentalmente por $\mathrm{SiO}_{2}, \mathrm{Al}_{2} \mathrm{O}_{3}, \mathrm{Fe}_{2} \mathrm{O}_{3}, \mathrm{CaO}, \mathrm{MgO}, \mathrm{Na}_{2} \mathrm{O}, \mathrm{K}_{2} \mathrm{O}$, encontrándose el resto de elementos químicos en mucha menor cantidad. Desde el punto de vista de la posible utilización de los residuos cocidos en la fabricación de clínker de cemento las diferencias en su composición química no son relevantes, aunque se puede destacar: a) el mayor contenido en hierro de los residuos cocidos R-R1, R-R2 y R-R3 en comparación con el de los residuos cocidos 
Residuos cerámicos para su posible uso como materia prima en la fabricación de clínker de cemento Portland: caracterización y activación alcalina

Ceramic wastes as raw materials in Portland cement clinker fabrication: characterization and alkaline activation

TABLA 2

Composición química de los residuos cocidos (porcentaje en peso)

\begin{tabular}{|c|c|c|c|c|c|c|}
\hline ÓXIDO & $R-R 1$ & $R-R 2$ & $R-R 3$ & $R-B 1$ & $R-B 2$ & $R-M$ \\
\hline $\mathrm{SiO}_{2}$ & 61.5 & 60.3 & 63.8 & 67.3 & 64.8 & 62.2 \\
\hline $\mathrm{Al}_{2} \mathrm{O}_{3}$ & 16.1 & 16.4 & 17.4 & 18.0 & 18.0 & 16.8 \\
\hline $\mathrm{Fe}_{2} \mathrm{O}_{3}$ & 5.3 & 5.32 & 3.55 & 1.10 & 1.82 & 4.7 \\
\hline $\mathrm{CaO}$ & 7.9 & 7.02 & 6.55 & 6.50 & 9.92 & 6.75 \\
\hline $\mathrm{MgO}$ & 2.35 & 3.07 & 1.90 & 0.64 & 0.42 & 2.71 \\
\hline $\mathrm{Na}_{2} \mathrm{O}$ & 0.77 & 0.65 & 1.27 & 1.72 & 0.62 & 0.81 \\
\hline $\mathrm{K}_{2} \mathrm{O}$ & 3.70 & 4.31 & 3.05 & 2.12 & 2.09 & 3.95 \\
\hline $\mathrm{TiO}_{2}$ & 0.68 & 0.68 & 0.61 & 0.50 & 0.70 & 0.67 \\
\hline $\mathrm{MnO}$ & 0.05 & 0.05 & 0.04 & 0.01 & 0.01 & 0.04 \\
\hline $\mathrm{P}_{2} \mathrm{O}_{5}$ & 0.16 & 0.21 & 0.23 & 0.11 & 0.21 & 0.17 \\
\hline $\mathrm{ZnO}$ & 0.33 & 0.52 & 0.35 & 0.69 & 0.42 & 0.36 \\
\hline $\mathrm{ZrO}_{2}$ & 0.42 & 0.42 & 0.50 & 0.45 & 0.31 & 0.32 \\
\hline $\mathrm{SO}_{3}$ & 0.07 & 0.06 & 0.06 & 0.05 & 0.13 & 0.05 \\
\hline $\mathrm{ppc}$ & 0.33 & 0.30 & 0.36 & 0.29 & 0.48 & 0.29 \\
\hline
\end{tabular}

TABLA 3

Composición química de los residuos cocidos $\left(\mathrm{mg} \cdot \mathrm{kg}^{-1}\right)$

\begin{tabular}{|c|c|c|c|c|c|c|}
\hline ÓxIDO & $R-R 1$ & $R-R 2$ & $R-R 3$ & $R-B 1$ & $R-B 2$ & $R-M$ \\
\hline $\mathrm{PbO}$ & 400 & 190 & 290 & 160 & 150 & 125 \\
\hline $\mathrm{BaO}$ & 1200 & 1300 & 1000 & 405 & 600 & 485 \\
\hline $\mathrm{B}_{2} \mathrm{O}_{3}$ & 1880 & 2029 & 1370 & 2145 & 1830 & 1835 \\
\hline $\mathrm{Cr}_{2} \mathrm{O}_{3}$ & 117 & 99 & 115 & 198 & 120 & 59 \\
\hline $\mathrm{V}_{2} \mathrm{O}_{5}$ & 125 & 134 & 129 & 88 & 118 & 150 \\
\hline $\mathrm{Co}_{3} \mathrm{O}_{4}$ & $<20$ & $<20$ & $<20$ & $<20$ & $<20$ & $<20$ \\
\hline $\mathrm{NiO}$ & $<20$ & $<20$ & $<20$ & $<20$ & $<20$ & $<20$ \\
\hline $\mathrm{CuO}$ & $<20$ & $<20$ & $<20$ & $<20$ & $<20$ & $<20$ \\
\hline $\mathrm{As}_{2} \mathrm{O}_{3}$ & $<20$ & $<20$ & $<20$ & $<20$ & $<20$ & $<20$ \\
\hline $\mathrm{Sb}_{2} \mathrm{O}_{3}$ & $<20$ & $<20$ & $<20$ & $<20$ & $<20$ & $<20$ \\
\hline $\mathrm{CdO}$ & $<20$ & $<20$ & $<20$ & $<20$ & $<20$ & $<20$ \\
\hline
\end{tabular}

$\mathrm{R}-\mathrm{B} 1$ y R-B2, como consecuencia de que los primeros proceden de productos fabricados con arcillas rojas mientras que los segundos proceden de productos fabricados con arcillas blancas; b) el contenido en hierro del residuo cocido R-M presenta un valor intermedio, consecuencia de que esta muestra es una mezcla de residuos cocidos procedentes tanto de productos fabricados con arcillas rojas como blancas; c) el contenido en $\mathrm{CaO}$ oscila alrededor del $7 \%$ en todos los residuos cocidos, excepto en el R-B2 que llega a ser de casi el $10 \%$. Por lo tanto, desde el punto de vista 
de la composición química los seis residuos cocidos son susceptibles de ser utilizados en la fabricación de clínker, aunque cuanto mayor sea su contenido en $\mathrm{CaO}$ más favorable será dicha utilización.

En la Tabla 4 se detallan las fases cristalinas y amorfas presentes en cada residuo cocido y su cantidad. Puede observarse que los seis residuos cocidos presentan las mismas fases aunque pueden variar sus proporciones. Se puede concluir que las fases, tanto las cristalinas como las amorfas, presentes en los residuos cocidos no impiden, sino todo lo contrario, su posible utilización en la fabricación de cementos. Algunos componentes mineralógicos presentes en los residuos, como la gelenita y la wollastonita, son fases intermedias que se forman durante el proceso de clinkerización. Su presencia podría acelerar los procesos reactivos, aspecto que es necesario confirmar en estudios posteriores.
Se deduce, por tanto, que desde el punto de vista químico y mineralógico todos los residuos son potencialmente aptos para su empleo como materia prima alternativa en la fabricación de clínker de cemento Portland.

En la Figura 1 se muestran las curvas de contracción-temperatura, correspondientes a los seis residuos cocidos, y en la Tabla 5 se detallan las temperaturas características. Puede observarse una clara diferencia en la fusión de los residuos cocidos $\mathrm{R}-\mathrm{R} 1, \mathrm{R}-\mathrm{R} 2$ y $\mathrm{R}-\mathrm{R} 3$ en comparación con la de los residuos cocidos R-B1 y R-B2, presentando los primeros una tendencia a la fusión siempre a temperaturas más bajas que los segundos. A la vista de la composición química y de las fases presentes en cada residuo cocido (Tablas 2 a 4) esta diferencia en la fusión se debe, entre otros factores, a la diferencia en contenido en $\mathrm{Fe}_{2} \mathrm{O}_{3}$ como consecuencia de que los residuos cocidos R-R1, R-R2 y R$\mathrm{R} 3$ proceden de productos fabricados con arcillas rojas,

TABLA 4

Composición de fases de los residuos cocidos (porcentaje en peso)

\begin{tabular}{|c|c|c|c|c|c|c|}
\hline FASE & R-R1 & R-R2 & R-R3 & R-B1 & R-B2 & R-M \\
\hline Cuarzo & 23 & 27 & 33 & 40 & 37 & 26 \\
\hline Anortita & 15 & 14 & 24 & 33 & 32 & 14 \\
\hline Feldespato potásico & 7 & 7 & 8 & 10 & 8 & 8 \\
\hline Albita & 3 & 3 & 4 & 10 & 5 & 3 \\
\hline Hematites & 4 & 3 & 3 & 1 & 1 & 3 \\
\hline Diópsido & 5 & 5 & 3 & $<1$ & $<1$ & 5 \\
\hline Gehlenita & 2 & 1 & 1 & 1 & 1 & 1 \\
\hline Circón & $<1$ & 1 & 1 & 1 & $<1$ & 1 \\
\hline Wollastonita & 4 & 4 & $<1$ & $<1$ & 6 & 4 \\
\hline Fase Amorfa & 36 & 36 & 24 & 5 & 9 & 34 \\
\hline
\end{tabular}

TABLA 5

Temperaturas características de las curvas de contracción-temperatura de los residuos $\operatorname{cocidos}\left({ }^{\circ} \mathrm{C}\right)$

\begin{tabular}{|c|c|c|c|c|c|c|}
\hline & $R-R 1$ & $R-R 2$ & $R-R 3$ & $R-B 1$ & $R-B 2$ & $R-M$ \\
\hline$T_{I C}$ & 1110 & 1110 & 1070 & 1090 & 1070 & 1030 \\
\hline$T_{F C}$ & 1220 & 1200 & 1200 & 1270 & 1240 & 1210 \\
\hline$T_{R}$ & 1260 & 1240 & 1260 & 1330 & 1320 & 1260 \\
\hline$T_{E}$ & 1290 & 1270 & 1290 & 1360 & 1350 & 1280 \\
\hline$T_{1 / 2}$ & 1320 & 1310 & 1360 & 1390 & 1380 & 1330 \\
\hline$T_{F}$ & 1340 & 1330 & 1380 & 1420 & 1410 & 1350 \\
\hline
\end{tabular}



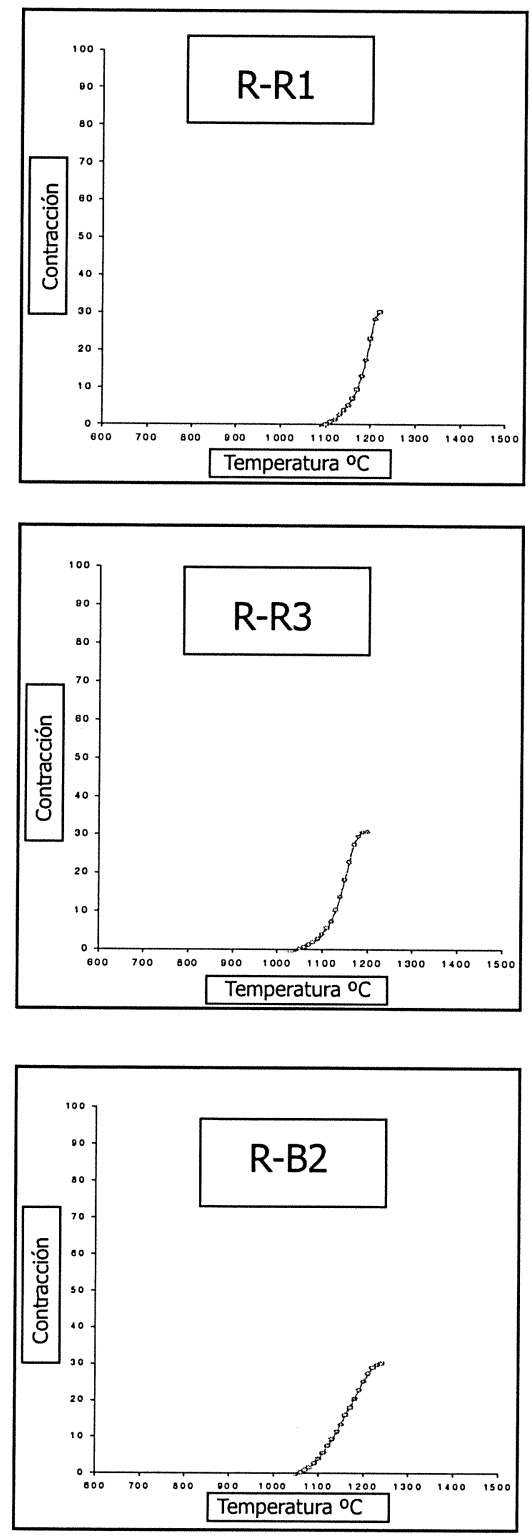

Figura 1.- Curva contracción-temperatura de los 6 residuos.

TABLA 6

Toxicidad de los residuos cocidos

\begin{tabular}{|c|c|c|c|c|c|c|}
\hline & $R-R 1$ & $R-R 2$ & $R-R 3$ & $R-B 1$ & $R-B 2$ & $R-M$ \\
\hline$E C_{50}\left(m q \cdot L^{-1}\right)$ & $226 \cdot 10^{3}$ & $82 \cdot 10^{3}$ & $199 \cdot 10^{3}$ & $98 \cdot 10^{3}$ & $90 \cdot 10^{3}$ & $191 \cdot 10^{3}$ \\
\hline
\end{tabular}

mientras que los residuos cocidos R-B1 y R-B2 proceden de productos fabricados con arcillas blancas. El proceso de fusión del residuo cocido R-M presenta, de nuevo, un comportamiento intermedio consecuencia de que esta muestra es una mezcla de residuos cocidos procedentes tanto de productos fabricados con arcillas rojas como blancas. Aunque las diferencias encontradas en las temperaturas de fusión no son muy significativas, sin embargo, desde el punto de vista de la posible utilización de los residuos cocidos en la fabricación de clínker de cemento, pueden tener importancia y afectar a la temperatura de formación y a las características físicas
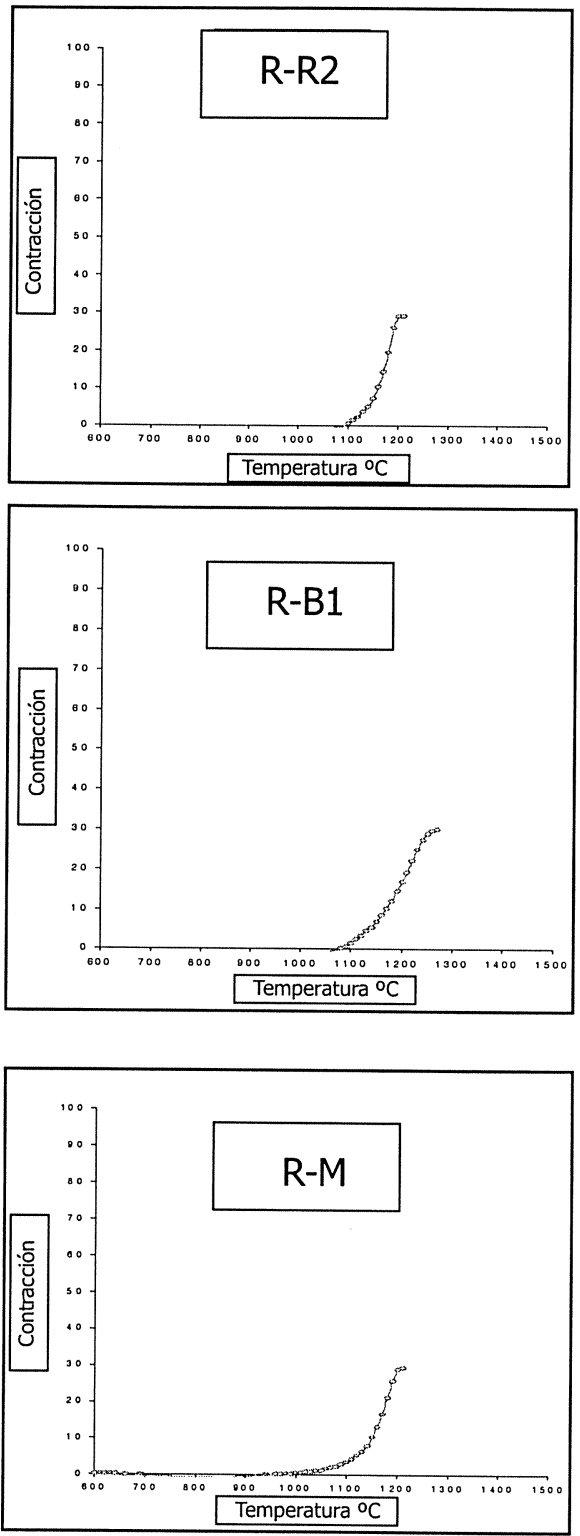
TABLA 7

Actividad reactiva $\left(\mathrm{Bq} \cdot \mathrm{kg}^{-1}\right)$ e índice de concentración de actividad de los residuos cocidos

\begin{tabular}{|c|c|c|c|c|c|c|}
\hline & R-R1 & R-R2 & R-R3 & R-B1 & R-B2 & R-M \\
\hline Ra-226 & $69 \pm 5$ & $67 \pm 5$ & $75 \pm 4$ & $81 \pm 6$ & $67 \pm 5$ & $69 \pm 5$ \\
\hline Th-232 & $68 \pm 11$ & $57 \pm 7$ & $65 \pm 7$ & $59 \pm 9$ & $70 \pm 10$ & $68 \pm 11$ \\
\hline K-40 & $1196 \pm 66$ & $1121 \pm 63$ & $997 \pm 44$ & $717 \pm 54$ & $622 \pm 49$ & $1197 \pm 66$ \\
\hline Índice & 0.97 & 0.88 & 0.91 & 0.80 & 0.78 & 0.94 \\
\hline
\end{tabular}
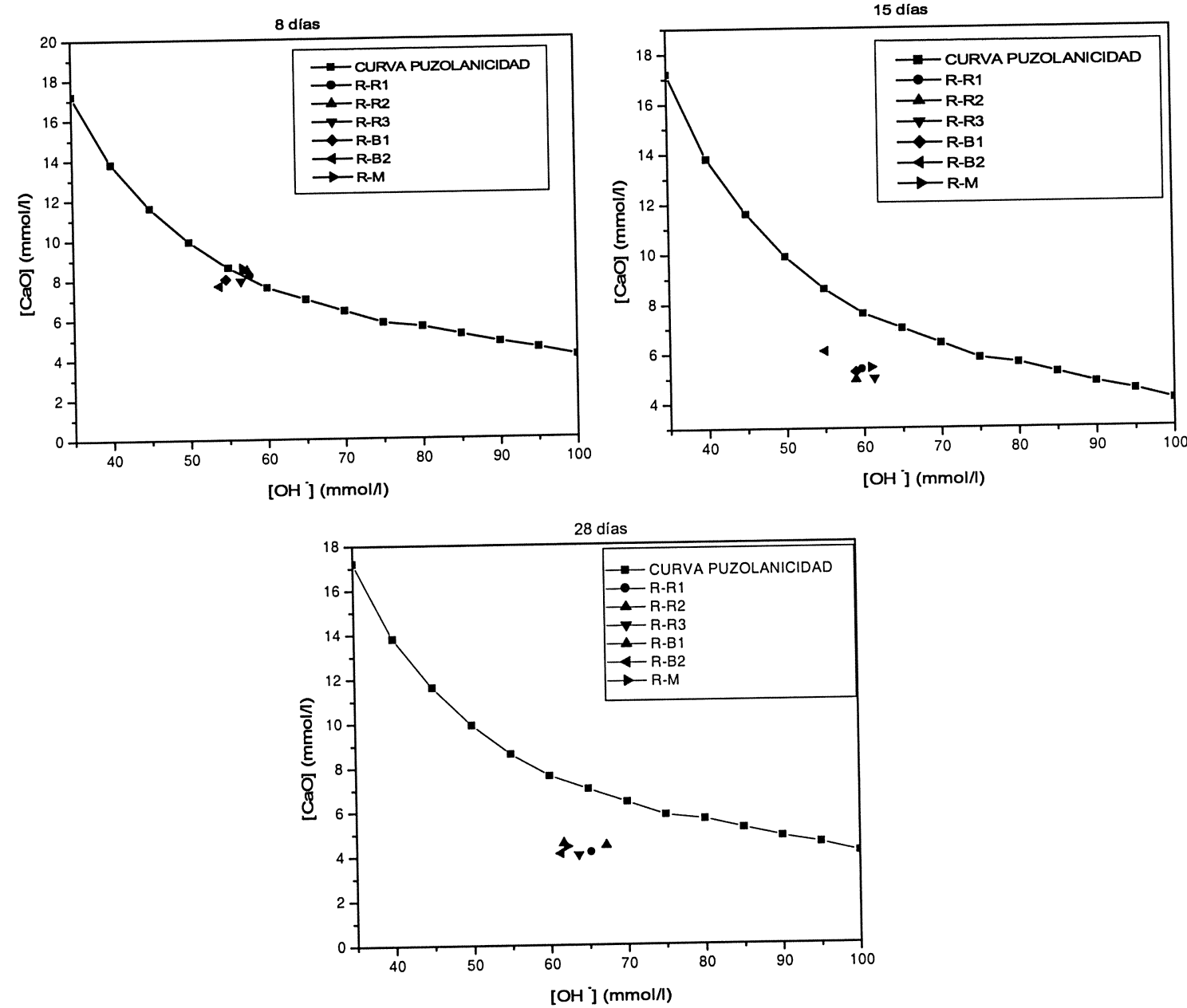

Figura 2.- Resultados del ensayo de puzolanicidad EN 196-5 a los 8, 15 y 28 días.

de 1989, sobre caracterización de materiales tóxicos y peligrosos, un material se considerará tóxico si presenta un valor de $\mathrm{EC}_{50}\left(15^{\circ} \mathrm{C}, 15 \mathrm{~min}\right)$ inferior a $3.000 \mathrm{mg} \cdot \mathrm{L}^{-1}$. En consecuencia, a la vista de los resultados obtenidos, se concluye que los residuos cocidos no son tóxicos según la legislación española vigente.

En la Tabla 7 se detalla la actividad de los isótopos Ra-226, Th-232 y K-40 y el índice de radioactividad para cada residuo cocido. Según la Unión Europea (42), un material de construcción se considerará radioactivo si presenta un índice de actividad igual o superior a 6 . En consecuencia, a la vista de los resultados, se concluye que los residuos cocidos se pueden considerar como no radioactivos.

En la Figura 2 se presenta la evolución de la actividad puzolánica de los residuos cerámicos estudiados de acuerdo con la norma EN 196-5 (43). Del análisis de estas gráficas se deduce que los residuos R-B1, R-B2 y R-R3 ya evidencian comportamiento puzolánico a los 8 días de 

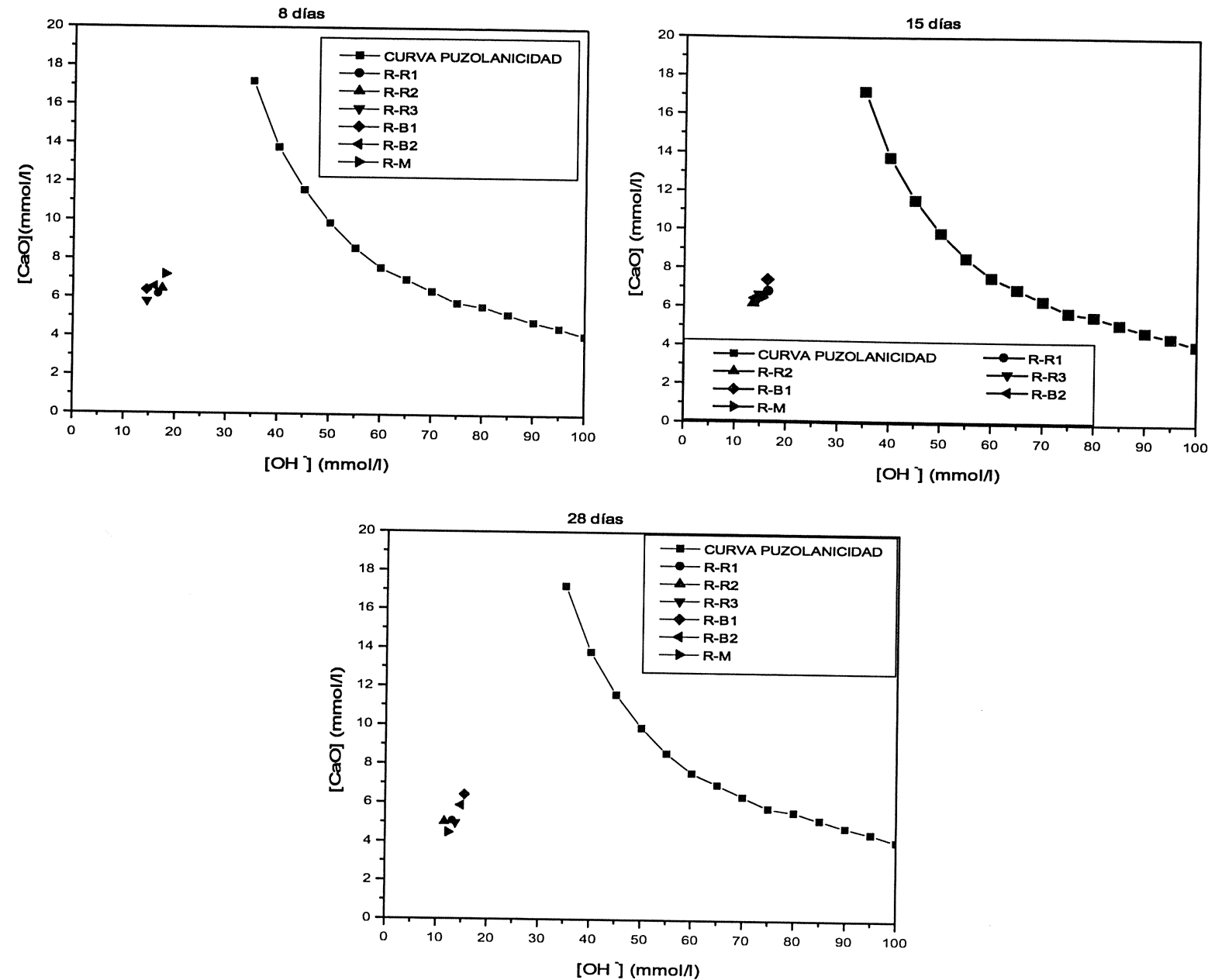
Figura 3.- Resultados de la determinación de la actividad puzolánica de los residuos en una disolución saturada de $\mathrm{Ca}(\mathrm{OH})_{2}$ a los 8 ,
15 y 28 días.

reacción, ya que las concentraciones de $\mathrm{CaO}$ y $[\mathrm{OH}]^{-}$se encuentran por debajo de la línea de saturación. Estos residuos son los que tienen el mayor contenido en $\mathrm{SiO}_{2} y$ $\mathrm{Al}_{2} \mathrm{O}_{3}$. A partir de los 15 días de ensayo todos los residuos tienen actividad puzolánica. La composición química (alto contenido en $\mathrm{SiO}_{2}$ y $\mathrm{Al}_{2} \mathrm{O}_{3}$ ), así como la composición mineralógica y el contenido en fase vítrea (superior en algunos casos al $30 \%$ ) de estos residuos explican este comportamiento. En la Figura 3 se muestran los resultados del ensayo con disolución saturada de $\mathrm{Ca}(\mathrm{OH})_{2}$. Dichos resultados confirman lo mostrado anteriormente, aunque en estas condiciones todos los residuos, desde los 8 días de tratamiento, presentan comportamiento puzolánico. Estos resultados obtenidos coinciden por los indicados por otros autores (44) con materiales de desecho procedentes de arcilla cocida.

Los resultados obtenidos tras los ensayos de activación alcalina de los residuos (Figura 4) demuestran, que a la edad de ensayo (8 días), la activación de los residuos es baja, no obteniéndose resistencias mecánicas a compresión y flexotracción superiores a los $13 \mathrm{MPa}$ y a $4 \mathrm{MPa}$, respectivamente. La naturaleza del activador no parece ser un factor determinante a diferencia de lo que ocurre en otros procesos de activación alcalina (46); aunque se obtienen ligeros incrementos en las resistencias cuando el activador alcalino es la disolución de waterglass. Finalmente, también se deduce que las mayores resistencias se obtienen cuando la concentración del activador es $6 \mathrm{M}$. El contenido en fase amorfa tampoco parece ser un factor determinante en la activación alcalina de los residuos. Los residuos R-R1, R-R2 y R-M tienen porcentajes de fase amorfa superiores al $34 \%$ (Tabla 4 ), y sus valores resistentes son del mismo orden de los obtenidos por los otros residuos con porcentajes de fase amorfa inferior al $10 \%$.

El análisis mineralógico realizado por DRX sobre las muestras activadas no ha aclarado el proceso de activación de los residuos. No se han observado en los difractogramas diferencias sustanciales que indiquen la formación de fases cristalinas como resultado de la activación, ni cuáles son las fases presentes en el residuo más susceptibles de reacción. Como consecuencia de la activación alcalina de este tipo de materiales se forman productos de muy baja cristalinidad y difícilmente detectables por DRX. 

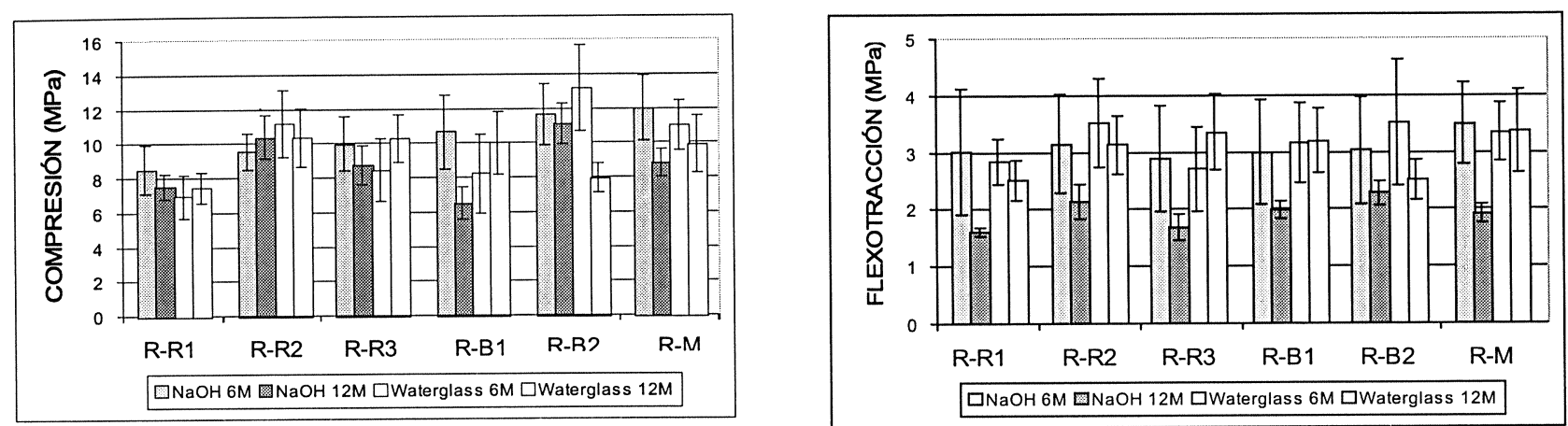

Figura 4.- Resistencias Mecánicas de las pastas activadas.

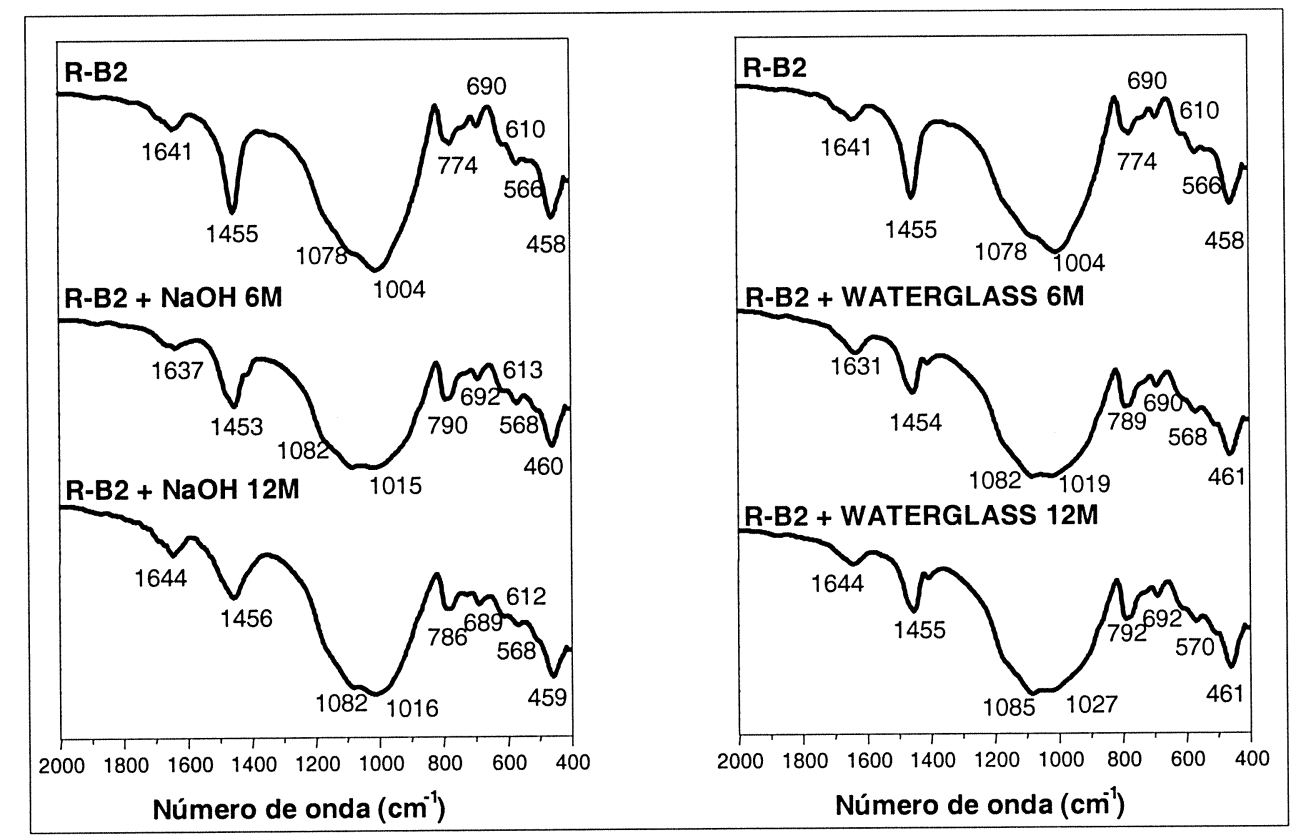

Figura 5.- FTIR del residuo R-B2 y sus pastas activadas.

La espectroscopia IR tampoco ha resultado ser una técnica determinante para definir el proceso de activación de estos residuos, pero sí ha dado alguna información de interés en torno a dicho proceso. En la Figura 5 se muestran los espectros IR del residuo R-B2 y sus pastas activadas (los restantes muestras tienen espectros IR similares). El espectro IR del residuo original presenta las bandas de absorción características de identificación del cuarzo y la anortita (47), con un máximo de absorción hacia 1.004 $\mathrm{cm}^{-1}$ debido a vibraciones $v_{3}$ (Si-O) muy probablemente de las fases feldespáticas (albita y feldespato potásico) (47). En los espectros IR de la pasta activada con $\mathrm{NaOH}$ $6 \mathrm{M}$, esta banda disminuye en intensidad, marcándose con mayor claridad las bandas identificativas del cuarzo y la anortita; es decir, parece que en estas condiciones de activación, las fases feldespáticas son las más reactivas. Este mismo fenómeno se observa en los espectros IR de las pastas activadas con $\mathrm{NaOH} 12 \mathrm{M}$ y waterglass con las dos concentraciones. A través de la sustracción de espectros IR se induce la posible formación de una nueva fase silicato, ya que aparece una nueva banda de vibración hacia $990 \mathrm{~cm}^{-1}$. Con otros residuos, se ha observado a través de FIIR, que ese posible fenómeno de disolución parcial de las fases feldespáticas se observa mucho más marcado cuando la disolución de $\mathrm{NaOH}$ es $6 \mathrm{M}$, que cuando es $12 \mathrm{M}$.

En la Figura 6 se muestran los espectros de ${ }^{29} \mathrm{Si}$ RMN, tanto del residuo R-B2 original como de su pasta activada con $\mathrm{NaOH} 6 \mathrm{M}$. Del análisis de estos espectros se deduce que aunque en ambos espectros las señales aparecen a los mismos valores de ppm, se produce una disminución en la anchura e intensidad de las mismas. De acuerdo a la bibliografía (48) la señal en -109 ppm se asigna a unidades $\mathrm{Q}^{4}$, la situada en -102 a unidades $\mathrm{Q}^{4}(1 \mathrm{Al})$, a $-98 \mathrm{ppm}$ a unidades $\mathrm{Q}^{4}(2 \mathrm{Al})$, a $-94 \mathrm{ppm}$ a unidades $\mathrm{Q}^{3}$, a $-89 \mathrm{ppm}$ a unidades $\mathrm{Q}^{3}(1 \mathrm{Al})$ y finalmente $a$ -84 a unidades $Q^{2}$. Todas estas señales son asignables a las fases mineralógicas detectadas por DRX y FTIR. La persistencia de las señales está confirmando que la activación de los residuos no es intensa, aunque el cambio significativo en la intensidad y anchura de las mismas está, a su vez, mostrando que algunas de esas fases (probablemente las feldespáticas) han reaccionado parcialmente. 


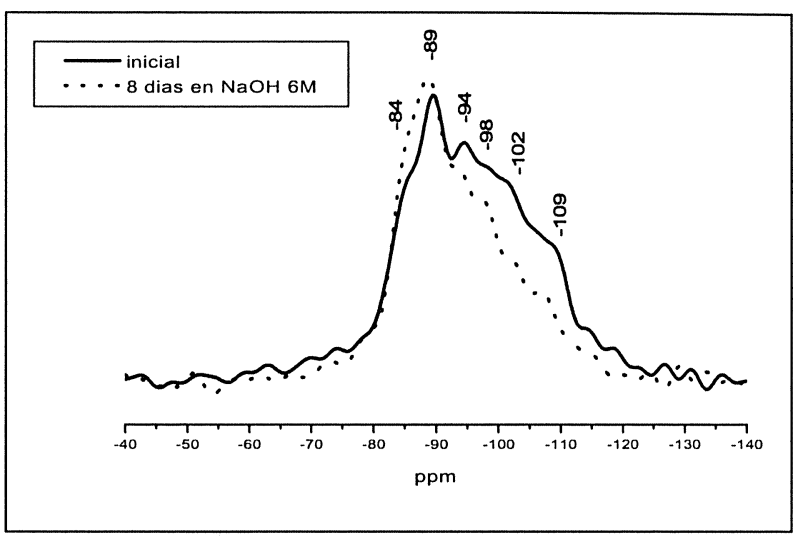

Figura 6.- ${ }^{29} \mathrm{Si}$ RMN del residuo R-B2 antes y después de activarlo con $\mathrm{NaOH} 6 \mathrm{M}$.

\section{CONCLUSIONES}

Tras el estudio de caracterización y activación alcalina realizado sobre los residuos cocidos, se concluye que:

a) Todos tienen una composición química y mineralógica adecuada para su posible empleo como materia prima alternativa de un crudo preparado para la fabricación de clínker de cemento Portland.

b) Se ha demostrado que los residuos cocidos estudiados no son tóxicos ni radioactivos.

c) Como se podía esperar, estos residuos tienen capacidad puzolánica, algunos de ellos desde los primeros 8 días de reacción. d) A la edad de estudio (8 días) la activación alcalina de los residuos es baja. Las resistencias mecánicas a compresión no superan los $14 \mathrm{MPa}$. La composición química y contenido en fase amorfa de los residuos no parece ser un factor determinante en la activación alcalina de los mismos.

La naturaleza del activador (disoluciones de $\mathrm{NaOH}$ o waterglass) tampoco parece ser un factor que influya en el proceso; obteniéndose las mayores resistencias cuando la concentración del activador es 6M. El estudio de caracterización realizado sobre las pastas activadas ha demostrado que las fases feldespáticas son las más susceptibles de disolución y reacción con las disoluciones alcalinas.

\section{AGRADECIMIENTOS}

Los autores agradecen al Ministerio de Educación y Ciencia (MEC) la subvención otorgada al proyecto CTM200406619-CO2-01 y 02/TECHNO dentro del cual se han realizado estas investigaciones.

M. Palacios también agradece a la Consejería de Educación de la Comunidad de Madrid la concesión de una beca predoctoral y S. Martínez-Ramírez al Ministerio de Educación y Ciencia y al ERDF la financiación del Contrato Ramón y Cajal.

Finalmente, los autores desean agradecer a M. M. Alonso, L. Ureña, P. Rivilla, A. Gil y J. L. García su colaboración en la realización de algunos de los ensayos.

\section{BIBLIOGRAFÍA}

(1) F. Rougemont, «Sustainable development in the cement industry: The South African record». 11th ICCC, Durban, 2003, pp. 9-18.

(2) F. Puertas, M. T. Blanco-Varela, «Empleo de combustibles alternativos en la fabricación de cemento. Efecto en las características y propiedades de clínkeres y cementos», Mater. Construcc., n 274 (2004), pp. 51-64.

(3) Norma UNE-ENV 197-1, 2002.

(4) F. Puertas, M. T. Blanco-Varela, A. Palomo, T. Vázquez, «Reactivity and burnability of raw mixes made with crystallized blastfurnace slag. Part I», Zement Kalk Gips, 8 (1988) pp. 398-402.

(5) F. Puertas, M. T. Blanco-Varela, A. Palomo, T. Vázquez, «Reactivity and burnability of raw mixes made with crystallized blastfurnace slag. Part I», Zement Kalk Gips, 12 (1988), pp. 628-631.

(6) F. Puertas, M. T. Blanco-Varela, A. Palomo, T. Vázquez, «Reactividad y aptitud a la cocción de crudos de cemento que contienen escorias cristalizadas de horno alto», Cemento y Hormigón, 854 (2003), pp. 4-10.

(7) A. Monshi, M. Kasiri, «Producing portland cement from iron and steel slags and limestone», Cem. Concr. Res, Vol. 29 (1999), pp. $1373-1377$.

(8) H. Hirao, S. Yokohama, «Behaviour of chloride ions in hardened ecoment: New type of Pórtland cement made from municipal waste incinerator ash». 11th ICCC, Durban (2003), pp. 1271-1281.

(9) P. H. Shih, J. E. Chang, L. C. Chiang, «Replacement of raw mix in cement production by municipal solid waste incineration ash», Cem. Concr. Res., Vol. 23 (2003), pp. 1831-1836.

(10) S. Goñi, A. Guerrero, M. A. Macías, R. Peña, E. Fernández-Escalante, «Empleo de materiales secundarios como materia prima de nuevos tipos de cementos», Mater. Construcc., no 263-254 (2001), pp. 71-84.

(11) M. T. Blanco-Varela, T. Vázquez, A. Palomo, «Utilización de cenizas volantes y mineralizadores como materia prima en la fabricación de cemento», Mater. Construcc., 188 (1983), pp. 45-53.

(12) M. T. Blanco-Varela, «Reactivity of fly-ashes when they are used as constituent of Portland cement raw mixes», TIZ-Fachberichte, 5 (1984), pp. 304-307.

(13) M. T. Blanco-Varela, F. Puertas, A. Palomo, T. Vázquez, P. Artola, I. Alfaro, «Aptitud a la cocción de crudos de cemento Portland usando Paval como materia prima», Cemento y Hormigón, 809 (2000), pp. 358-377.

(14) F. Puertas, M. T. Blanco-Varela, T. Vázquez, «Behaviour of cement mortars containing an industrial waste from aluminium refining. Stability in $\mathrm{Ca}(\mathrm{OH})_{2}$ solutions», Cem. Concr. Res., Vol. 29 (1999), pp. 1673-1680.

(15) M. Singh, S. N. Upadhayay, P.M. Prasad, «Preparation of special cements from red mud», Waste Management, 16 (1996), pp. $665-670$.

(16) A. Diouri, A. Boukhari, J. Aride, F. Puertas, T. Vázquez, «Stable $\mathrm{Ca}_{2} \mathrm{SiO}_{3}$ solid solutio containing manganeso and phosphorus», Cem. Concr. Res., Vol. 27 (1997), pp. 1203-1212. 
(17) A. Diouri, A. Boukhari, J. Aride, F. Puertas, T. Vázquez, «Elaboración de un clínker belítico fosfático con L-C ${ }_{2} S-$ Estudio de su actividad hidráulica», Mater. Construcc., 249 (1998), pp. 23-32.

(18) M. Singh, M. Garg, «Making of anhydrite cement from waste gypsum», Cem. Concr. Res., Vol. 30 (2000), pp. 571-577.

(19) A. Hauser, U. Eggenberger, T, Mumenthaler, «Fly ash from cellulose industry as secondary raw material in autoclaved aerated concrete», Cem. Concr. Res., Vol. 29 (1999), pp. 297-302.

(20) J. E. Enrique, E. Monfort, «Situación actual y perspectivas de futuro de los residuos de la industria azulejera», Cerámica información, 221 (1996), pp. 20-34.

(21) J. E. Enrique, E. Monfort, G. Busani et. al., Tratamiento de emisiones gaseosas, efluentes líquidos y residuos sólidos de la industria cerámica, Instituto de Tecnología Cerámica-AICE, Castellón, 1992.

(22) E. Monfort, J. E. Enrique, M. F. Gazulla et. al., «Caracterización de residuos de la industria azulejera», Técnica Cerámica, 222 (1994), pp. 238-246.

(23) E. Criado, E. Sánchez, M. Regueiro, «All tiled up: Spanish ceramics and glass», Ind. Miner., 444 (2004), pp. 48-55.

(24) E. Criado, E. Sánchez, M. Regueiro, «La industria cerámica española, ¿ante un cambio de ciclo?», Bol. Soc. Esp. Ceram. Vidr., 43

(2004), pp. 85-101.

(25) E. Monfort, M. Monzò, J. C. Jarque et. al., «Recycling red-fired tile scrap in red-firing floor and wall tile compositions», Tile Brick Int., 16 (2000), pp. 420-427.

(26) E. Monfort, M. Monzò, J. C. Jarque et. al., «Reciclado de tiesto en composiciones de pavimento y revestimiento rojo (I)», Técnica Cerámica, 292 (2001), pp. 450-458.

(27) E. Monfort, M. Monzò, J. C. Jarque et. al., «Reciclado de tiesto en composiciones de pavimento y revestimiento rojo (II)», Técnica Cerámica, 293 (2001), pp. 629-633.

(28) J. E. Enrique, E. Monfort, J. V. Agramunt et. al., «Gestión de vertidos en la fabricación de baldosas cerámicas», Técnica Cerámica, 246 (1996), pp. 478-485.

(29) M. H. Young, K. K. Hyng, «A research on the recycling of ceramic wastes as an aggregate for concrete», Chawon Rissaikuring, 10 (2001), pp. 41-49.

(30) M. I. Sánchez-Rojas, M. Frías, J. Rivera et. al., «Waste products from prefabricated ceramic materials as pozzolanic addition». $11^{\text {th }}$ ICCC, Durban, 2003, pp. 935-943.

(31) M. I. Sánchez-Rojas, M. Frías, J. Rivera, «Study on the pozzolanic and calorimetric behaviour of industrial products and wastes for use as calcined natural pozzolans in the manufacture of portlandcement CEM/A and B-Q», Cemento y Hormigón, 837 (2002), pp. 12-21.

(32) Ay N, Ü. M., «The use of waste ceramic tile in cement production», Cem. Concr. Res., Vol. 30 (2000), pp. 497-499.

(33) A. Barba, M. F. Gazulla, M. P. Gómez et al., «Chemical characterization of geological raw materials used in traditional ceramics», Geostandards and Geoanalytical Research, 28 (2004), pp. 203-212.

(34) A. Barba, M. F. Gazulla, M. P. Gómez et al., «A methodology for characterizing ceramic wastes: management of Environmental Quality», An International Journal, 14 (2003), pp. 333-343.

(35) A. Barba, M.F. Gazulla, M.P. Gómez et al., «Preparation of reference materials for frit chemical analysis», Glastech. Ber.-Glass Sci. Technol, 75 (2002), pp. 184-190.

(36) A. Barba, M. F. Gazulla, M. P. Gómez et al., «Characterization of chromium-containing ceramic pigments by XRF and XRD», X-ray Spectrometry, 33 (2004), pp. 431-438.

(37) A. Barba, M. F. Gazulla, M. P. Gómez et al., «Characterisation of ceramic oxide refractories by XRF and XRD», X-ray Spectrometry, 33 (2004), pp. 421-430.

(38) A. Barba, M. F. Gazulla, M. P. Gómez et al., «Physico-chemical characterisation of silicon carbide refractories», J. Europ. Ceram. Soc., (in press).

(39) A. Barba, S. Maestre, E. Bou. et al., «Using the hot stage microscope for estimating frit sealing temperature», Cfi (Ceramic Forum International)/Ber. DKG, 78 (2001), E36-E39.

(40) Orden Ministerial del 13 de octubre de 1989, apéndice IV (BOE n 270, de 10 de noviembre de 1989).

(41) UNE-EN ISO 11348.

(42) Documento de la Unión Europea: «RP112 Radiological protection principles concerning the natural radioactivity of building materials».

(43) EN 196-5, «Métodos de ensayos de cementos. Ensayo de puzolanicidad para cementos puzolánicos».

(44) M. I. Sánchez de Rojas, M. Frías, J. Rivera et al., «Investigación sobre la reactividad puzolánica de materiales de desecho procedentes de arcilla cocida», Mater. Construcc., 262 (2001).

(45) A. Palomo, A. Fernández-Jiménez, M. Criado, «Geopolímeros: Una única base química y diferentes microestructuras», Mater. Construcc., 275 (2004), pp. 77-91.

(46) A. Fernández-Jiménez, J. G. Palomo, F. Puertas, «Alkali-activated slag mortars: mechanical strength behaviour», Cem. Concr. Res., Vol. 29 (1999), pp. 1313-1321.

(47) H. W. van der Marel, H. Beutelspacher, Atlas of Infrared spectroscopy of clay minerals and their admixtures, Ed Elsevier, 1976.

(48) G. Engelhardt, D. Michel, High resolution solid-state of silicates and zeolites, Ed. John Wiley and Sons, 1987. 